\title{
OSTEOSSÍNTESE DAS FRATURAS DA EXTREMIDADE PROXIMAL DO ÚMERO COM SISTEMA DE PLACA DE ÂNGULO FIXO COM PARAFUSOS BLOQUEADOS: TÉCNICA E RESULTADOS
}

\author{
OSTEOSYNTHESIS OF PROXIMAL HUMERAL END FRACTURES WITH FIXED \\ ANGLE PLATE AND LOCKING SCREWS: TECHNIQUE AND RESULTS
}

Marcio Cohen', Marcus Vinicius Amaral', Martim Monteiro', Bruno Lobo Brandão', Geraldo Rocha Motta Filho²

\section{RESUMO}

Objetivo: Descrever os resultados do tratamento cirúrgico das fraturas da extremidade proximal do úmero com a placa de sistema bloqueado Philos. Métodos: Entre março de 2003 e outubro de 2004 foram avaliados, de forma prospectiva, 24 de 26 pacientes com fratura da extremidade proximal do úmero tratados com a placa Philos. A média de seguimento foi de 12 meses e da idade dos pacientes, de 57 anos. Seis pacientes tinham fraturas em quatro partes, 11 em três partes e nove em duas partes. A avaliação clínica foi feita por meio dos critérios da University of California at Los Angeles (UCLA). Resultados: A média do escore da UCLA foi de 30 pontos (17-35). Todas as fraturas consolidaram. Em três pacientes a fratura consolidou em varo. Nesses, o índice da UCLA médio foi de 27 pontos. Conclusão: A osteossíntese com a placa Philos permite fixação estável com bom resultado funcional.

Descritores - Fraturas do úmero; Fixação interna de fraturas; Estudos de coortes; Estudos prospectivos.

\begin{abstract}
Objective: Describe the results of proximal humeral fractures surgically treated with the Philos locking plate system. Method: Between March 2003 and October 2004 we prospectively reviewed 24 of 26 patients with proximal humerus fractures treated with a Philos plate. The mean follow-up time was 12 months and the mean age of patients was 57 years. Six patients had four-part proximal humerus fractures, 11 patients had three-part proximal humerus fractures, and nine patients had two-part proximal humerus fractures. Clinical evaluation was performed using the University of California at Los Angeles (UCLA) criteria. Results: The mean UCLA score was 30 points (17-34). All fractures showed union. Three patients showed fracture union at varus position. The mean UCLA score for these patients was 27 points. Conclusion: Osteosynthesis with Philos plate provides a stable fixation method with good functional outcome.
\end{abstract}

Keywords - Humeral fractures; Fracture fixation, Internal; Cohort studies; Prospective studies.

1 - Médico Assistente do Grupo de Ombro e Cotovelo do Instituto Nacional de Traumatologia e Ortopedia (INTO) - RJ.

2 - Mestre em Ortopedia pela Escola Paulista de Medicina/EPM-Unifesp; Diretor do Instituto Nacional de Traumatologia e Ortopedia (INTO)_ - RJ.

Trabalho realizado no Instituto Nacional de Traumatologia e Ortopedia (INTO-RJ).

Correspondência: Rua Washington Luiz, 47 - 20230-024 - Rio de Janeiro, RJ. E-mail: mcohen5@hotmail.com 


\section{INTRODUÇÃO}

A incidência de fraturas da extremidade proximal do úmero corresponde a 4 a $5 \%$ do total das fraturas ${ }^{(1)}$. São mais frequentes nos idosos; nos jovens, geralmente relacionam-se com trauma de alta energia ${ }^{(2)}$.

A maioria dessas fraturas é estável e com mínimo desvio, sendo passíveis de tratamento incruento. Entretanto, em 15 e $20 \%$ dos casos há necessidade de intervenção cirúrgica. Sempre que possível, a opção pela osteossíntese é empregada, visto que os resultados funcionais da hemiartroplastia não são suficientemente satisfatórios em grande parte dos $\operatorname{casos}^{(2)}$. O objetivo da osteossíntese é promover estabilidade que permita a mobilização precoce, além de se obter bom posicionamento dos fragmentos fraturados ${ }^{(2)}$.

A escolha do tipo de redução ou do material de síntese a ser empregado depende do padrão da fratura, da qualidade óssea, da idade e do grau de atividade do paciente $^{(2,3)}$. A utilização de placa e parafuso como método de fixação é uma opção difundida no meio ortopédico. No entanto, qualquer método está sujeito a complicações, como a soltura dos elementos de fixação, principalmente em pacientes mais idosos ${ }^{(3)}$.

Diante disso, a placa de ângulo fixo bloqueada foi desenvolvida para permitir fixação mais estável principalmente em ossos de má qualidade. A placa Philos (Proximal Humeral Internal Locking System) é um exemplo de implante dessa nova geração de placas com sistema bloqueado; é considerado implante de ângulo fixo, cujo desenho foi baseado na anatomia da região proximal do úmero ${ }^{(4)}$.

O objetivo deste estudo foi descrever a técnica de utilização da placa de ângulo fixo com parafusos bloqueados Philos e avaliar os resultados em 26 pacientes com lesões traumáticas da extremidade proximal do úmero submetidos a osteossíntese, em que foi empregado esse tipo de implante.

\section{MÉTODOS}

No período compreendido entre março de 2003 e outubro de 2004 foi realizado um estudo de coorte prospectivo, no qual 26 pacientes, sendo 14 do sexo feminino e 12 do masculino, foram tratados cirurgicamente pelo Grupo de Ombro e Cotovelo do Instituto Nacional de Traumatologia e Ortopedia (INTO-RJ), devido à fratura da extremidade proximal do úmero. Utilizou-se como método de fixação a placa de ângulo fixo com pa- rafusos bloqueados Philos para a extremidade proximal do úmero. Foram incluídos todos os casos de fraturas da extremidade proximal do úmero com indicação de osteossíntese tratados nesse período.

No período pré-operatório, foi preenchido um protocolo identificando-se: idade, sexo, mecanismo de trauma, presença de lesões associadas e a classificação da fratura segundo Neer e segundo a AO (Arbeitsgemeinschaft für Osteosynthesefrägen). A média de idade dos pacientes foi de 57 anos (24-85 anos). O mecanismo de fratura foi queda em 17 pacientes, acidente automobilístico em cinco e, nos demais, quatro causas variadas. Nos pacientes com menos de 50 anos (11), a causa de fratura mais comum foi o acidente automobilístico. De acordo com a classificação de Neer, nove fraturas foram classificadas em duas partes, 11 em três partes, sendo duas associadas à luxação anterior (Figura 1), e seis em
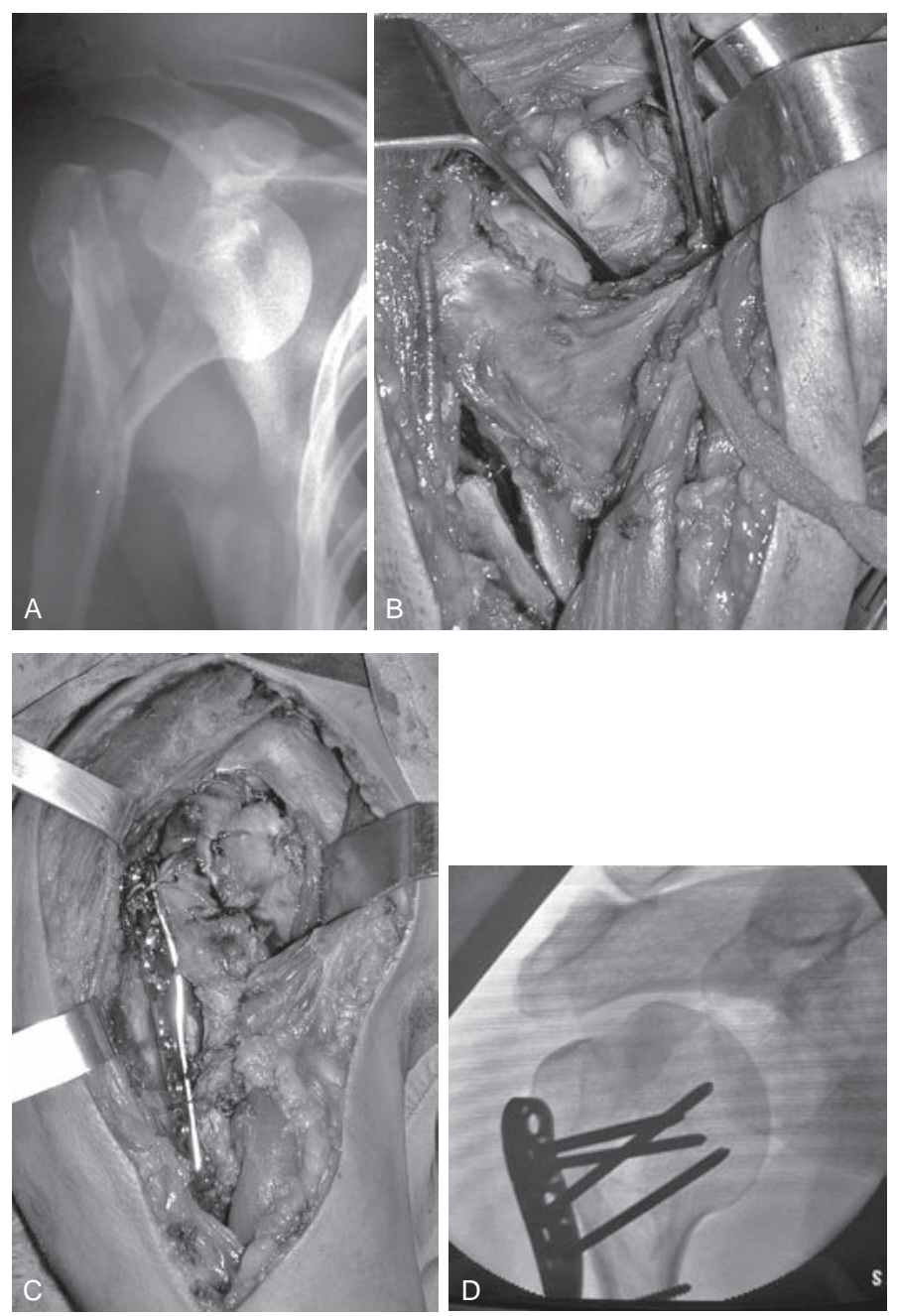

Figura 1 - A) Radiografia demonstrando fratura luxação anterior em três partes. (B e C) Aspecto per-operatório da fratura luxação e após redução e osteossíntese com placa Philos. D) Imagem fluoroscópica per-operatória. 
quatro partes, sendo quatro destas do tipo impactada em valgo (Tabela 1). Entre as lesões associadas houve um caso de fratura dos ossos da pelve, um caso de fratura da extremidade distal do rádio e um caso de fratura dos ossos da face. Preenchidos os critérios para indicação cirúrgica, foi realizada a osteossíntese utilizando-se a placa de ângulo fixo com parafusos bloqueados Philos (Figura 2). Os pacientes foram acompanhados em caráter ambulatorial com exame clínico e radiográfico (Figura 3).

Foram avaliadas a amplitude de movimento do ombro, a consolidação da fratura, a satisfação do paciente e a presença de complicações relacionadas com a técnica cirúrgica ou ao implante. A avaliação clínica foi realizada pelo sistema de pontos definido pela University of California at Los Angeles (UCLA) ${ }^{(5)}$.

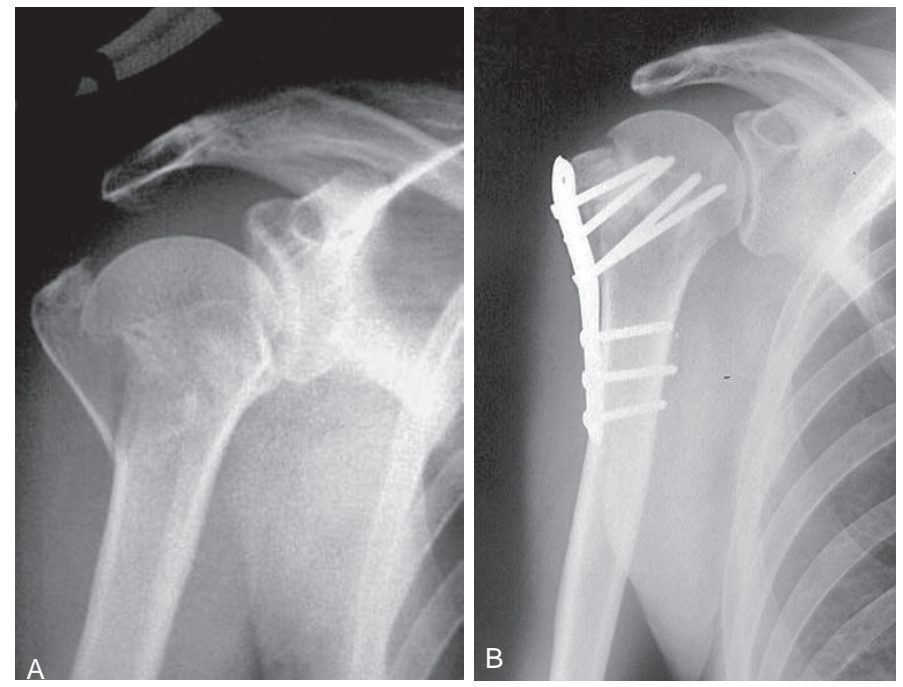

Figura 2 - A) Radiografia do ombro demonstrando fratura em quatro partes com desvio em valgo. B) Boa redução e fratura consolidada após osteossintese.

Tabela 1 - Relação dos pacientes

\begin{tabular}{|c|c|c|c|c|c|c|c|c|c|}
\hline Pac & Sexo & Idade & $\begin{array}{l}\text { Classificação } \\
\text { (Neer) }\end{array}$ & Classificação (AO) & Desvio pós & FA & RE & RI & UCLA pós \\
\hline 1 & M & 56 & 3 & B2 & NÃO & 110 & 40 & T8 & 29 \\
\hline 2 & $\mathrm{~F}$ & 85 & 2 & A3 & VARO & 90 & 30 & $\mathrm{~T} 10$ & 26 \\
\hline 3 & $\mathrm{~F}$ & 59 & 4 (imp valgo) & $\mathrm{C} 1$ & NÃO & 135 & 35 & T11 & 31 \\
\hline 4 & $M$ & 46 & 3 & B1 & VARO & 150 & 30 & $\mathrm{~T} 10$ & 29 \\
\hline 5 & M & 50 & 4 (imp valgo) & $\mathrm{C} 2$ & NÃO & 170 & 50 & T8 & 35 \\
\hline 6 & $\mathrm{~F}$ & 78 & 4 (imp valgo) & $\mathrm{C} 2$ & NÃO & 100 & 30 & $\mathrm{~T} 12$ & 26 \\
\hline 7 & M & 51 & 3 & B2 & NÃO & 120 & 30 & $\mathrm{~T} 10$ & 29 \\
\hline 8 & $\mathrm{~F}$ & 80 & 2 & A3 & VARO & 90 & 25 & L1 & 26 \\
\hline 9 & $\mathrm{~F}$ & 41 & 3 & B1 & NÃO & 170 & 50 & T8 & 33 \\
\hline 10 & $\mathrm{~F}$ & 74 & 2 & A3 & NÃO & 140 & 40 & T9 & 33 \\
\hline 11 & M & 61 & 4 (imp valgo) & $\mathrm{C} 2$ & NÃO & 160 & 40 & $\mathrm{~T} 10$ & 33 \\
\hline 12 & $\mathrm{~F}$ & 67 & 3 & B2 & NÃO & 130 & 40 & T12 & 30 \\
\hline 13 & $F$ & 34 & 4 & $\mathrm{C} 2$ & NÃO & 160 & 50 & T8 & 34 \\
\hline 14 & $M$ & 38 & 3 & B3 & NÃO & 150 & 45 & T9 & 31 \\
\hline 15 & $M$ & 43 & 2 & A3 & NÃO & 170 & 50 & T5 & 28 \\
\hline 16 & $F$ & 82 & 2 & A2 & NÃO & 150 & 45 & $\mathrm{~T} 10$ & 34 \\
\hline 17 & $F$ & 51 & 3 & B1 & NÃO & 140 & 30 & T8 & 25 \\
\hline 18 & $\mathrm{M}$ & 39 & 2 & A3 & NÃO & 150 & 45 & T6 & 30 \\
\hline 19 & $F$ & 61 & 3 & B1 & NÃO & 120 & 25 & T9 & 30 \\
\hline 20 & $M$ & 38 & 2 & B3 & NÃO & 60 & 50 & T11 & 17 \\
\hline 21 & $F$ & 44 & 3 & B1 & NÃO & 140 & 50 & T9 & 30 \\
\hline 22 & $M$ & 41 & 4 & $\mathrm{C} 2$ & NÃO & 130 & 30 & T9 & 30 \\
\hline 23 & $F$ & 74 & 2 & A3 & NÃO & 140 & 50 & T8 & 34 \\
\hline 24 & $\mathrm{M}$ & 24 & 3 & B2 & NÃO & 180 & 60 & T8 & 34 \\
\hline
\end{tabular}

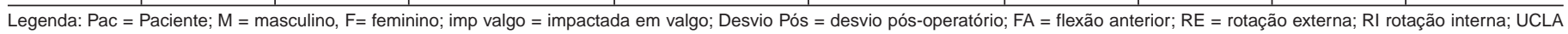
pós = UCLA pós-operatório.

Fonte: Instituto Nacional de Traumatologia e Ortopedia (INTO) 

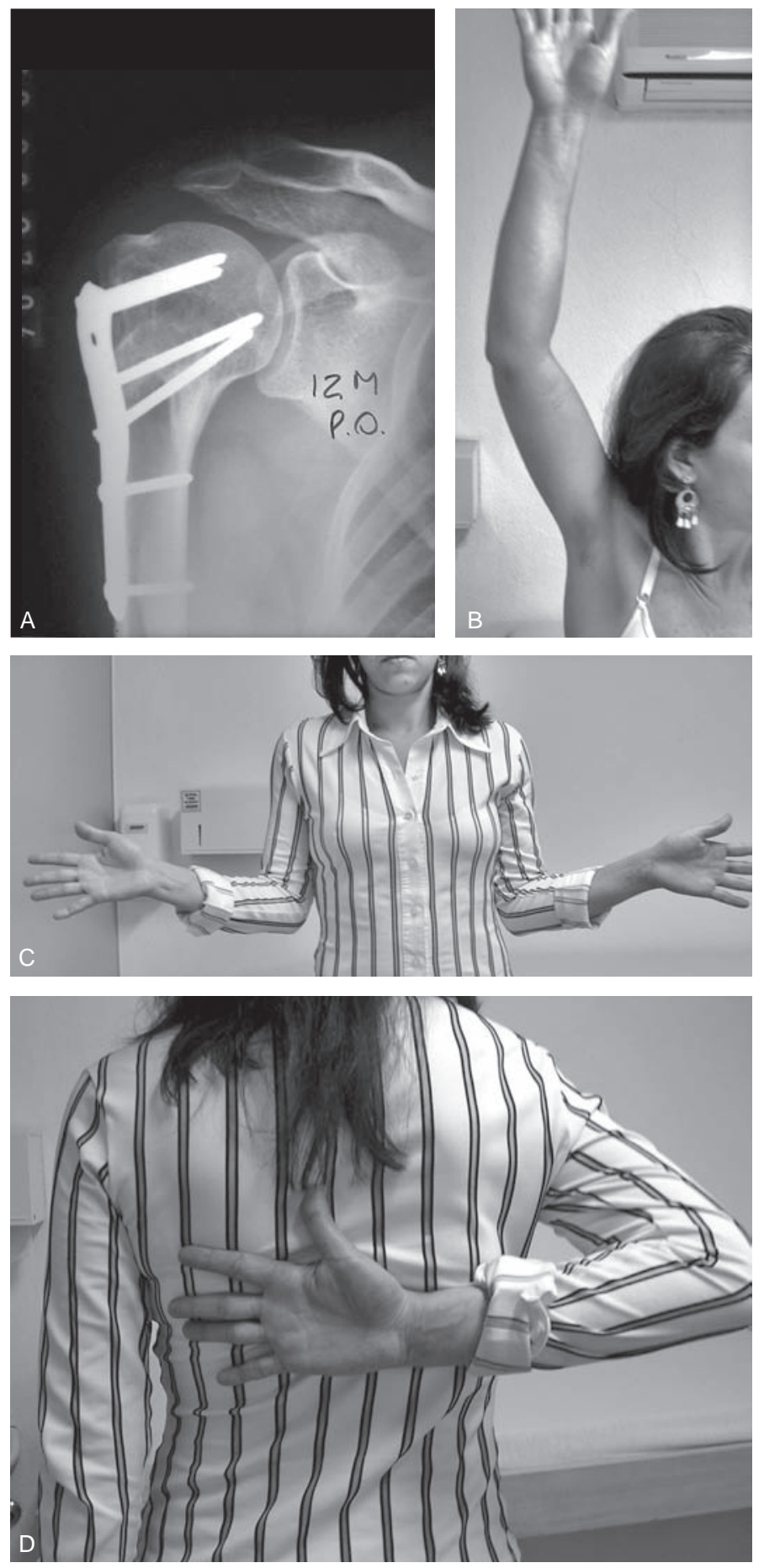

Figura 3 - A) Radiografia pós-operatória de fratura tratada cirurgicamente com placa Philos. B, C e D) Resultado funcional após 1 ano de cirurgia.

\section{TÉCNICA CIRÚRGICA}

Todas as cirurgias foram realizadas sob anestesia geral e com bloqueio escalênico para analgesia pós-operatória. A antibioticoterapia profilática (cefalosporina) iniciou-se com as induções anestésicas, perdurando nas 24 horas seguintes às do procedimento cirúrgico. Os pacientes foram posicionados em decúbito dorsal, "em cadeira de praia”, em mesa específica para cirurgia do ombro, o que permitiu a realização das três incidências radiográficas indispensáveis ao procedimento, sendo essas: ânteroposterior, de perfil e axilar do ombro. O intensificador de imagem foi colocado na cabeceira da mesa cirúrgica para que o arco pudesse girar livremente.

O acesso realizado foi o deltopeitoral. Como o posicionamento da placa determina a posição dos parafusos na cabeça do úmero, sempre que possível posicionávamos o implante de maneira que os parafusos se localizassem principalmente nas regiões central, inferior e posterior da cabeça umeral. O número de parafusos colocados na cabeça umeral foi em média de cinco (três a seis parafusos). No período pós-operatório imediato, o membro superior foi mantido na tipoia, iniciando-se a mobilização após a primeira visita ambulatorial entre o quinto e o sétimo dias.

\section{RESULTADOS}

Dos 26 pacientes incluídos, 24 retornaram para avaliação com seguimento médio de 12 meses (variando de oito a 24 meses). Todas as fraturas consolidaram com comprovação clínica e radiográfica.

O tempo decorrido entre a fratura e a osteossíntese foi em média de 14,4 dias (variando de dois a 50 dias). Sete pacientes apresentaram intervalo entre o trauma e a cirurgia maior do que 21 dias.

O resultado funcional avaliado pelo protocolo da UCLA apresentou escore médio de 30 pontos, variando de 17 a 35. Os pacientes apresentaram flexão anterior ativa, que variou de $60^{\circ}$ a $180^{\circ}$ (média de $135^{\circ}$ ), rotação lateral de $25^{\circ}$ a $60^{\circ}$ (média de $41^{\circ}$ ) e rotação medial de T8 a L1 (média de T9).

Três pacientes (11\%) apresentaram consolidação com angulação em varo (Figura 4); o escore funcional da UCLA desses pacientes foi em média de 27 pontos. Quando avaliados de forma isolada esses três pacientes, a flexão anterior ativa média foi de $110^{\circ}$; a rotação lateral média, de $28^{\circ}$; e a rotação medial média, de T11. Não foram observadas complicações como necrose avascular, lesão nervosa ou infecção. O paciente que apresentou fratura dos ossos da pelve evoluiu com trombose venosa profunda de membro inferior, que foi tratada de forma medicamentosa.

Todos os pacientes relataram satisfação com o resultado final e 20 (83\%) retornaram às atividades laborativas com o membro acometido. 

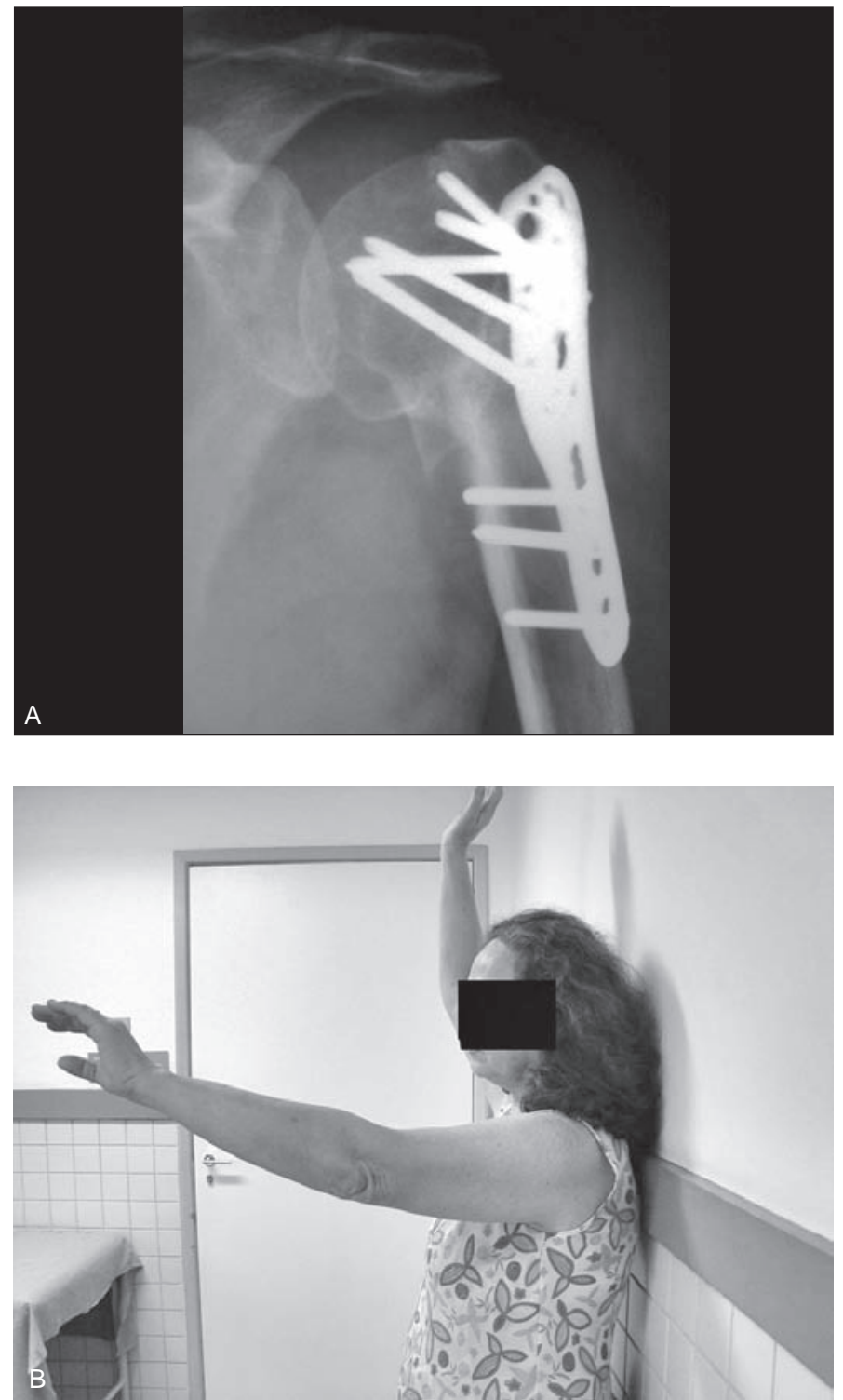

Figura 4 - A) Radiografia do ombro demonstrando fratura consolidada com desvio em varo. B) Paciente apresentando limitação da amplitude do arco de movimento do ombro.

\section{DISCUSSÃO}

Diversas técnicas estão descritas para a fixação das fraturas da extremidade proximal do úmero, como a utilização de fio de cerclagem, banda de tensão, fio de Kirschner, placa em “T”, haste intramedular, placa-lâmina e, mais recentemente, as placas bloqueadas de ângulo fixo, tendo cada um desses métodos suas vantagens e desvantagens $^{(6,7)}$.

Métodos de fixação menos invasivos, como a fixação percutânea descrita por Braman e Flatow ${ }^{(8)}$ são atraentes, porém, tecnicamente difíceis, principalmente em pacientes com osteoporose, nas fraturas com grande cominuição óssea e nas muito desviada.
O principal objetivo da fixação da fratura da extremidade proximal do úmero é a obtenção de construção estável que permita reabilitação precoce. Koval et $\mathrm{al}^{(9)}$ demonstraram que, em pacientes com fraturas minimamente desviadas, tratadas incruentamente, foram obtidos melhores resultados funcionais nos que iniciaram a reabilitação em até duas semanas após a fratura.

Alguns autores apresentam resultados satisfatórios com a osteossíntese com placas convencionais. Wanner et $\mathrm{al}^{(10)}$ descreveram bons resultados utilizando duas placas terço de cano. Porém, em ossos osteopênicos e nas fraturas cominutivas, a fixação com placas convencionais pode apresentar alta incidência de complicações, principalmente a soltura do material de síntese ${ }^{(11)}$.

Kristiansen e Christensen ${ }^{(11)}$ relataram apenas nove resultados satisfatórios em 20 pacientes (média de idade de 63 anos) tratados com a placa em "T", para fratura proximal do úmero, com alta incidência de falha de fixação.

Hintermann et $\mathrm{al}^{(12)}$ descreveram bons resultados em fraturas fixadas com placa-lâmina. Porém, recentemente, Meier et $\mathrm{al}^{(13)}$ relataram 33\% de complicações com esse método; em $22 \%$ dos pacientes houve protrusão da placa-lâmina na articulação do ombro.

Siffri et al ${ }^{(14)}$, em estudo biomecânico em modelo cadavérico, demonstraram maior rigidez da placa bloqueada nas forças torsionais quando comparada com a da placa-lâmina. Edwards et al $^{(15)}$ demonstraram também, em estudo cadavérico, que a resistência biomecânica da placa bloqueada, tanto a força de angulação quanto a de torsão, em fraturas cominutas do colo cirúrgico, é maior quando comparada com a da haste intramedular bloqueada.

As placas bloqueadas de ângulo fixo foram desenvolvidas para preencher os requisitos de estabilidade, principalmente em ossos osteopênicos, sem aumentar os riscos de osteonecrose. Existem poucos trabalhos na literatura com a utilização da placa Philos para essas fraturas.

Em estudo recente, Bjorkenheim et $\mathrm{al}^{(16)}$, utilizando-se dessa placa, relataram bons resultados em 72 pacientes com seguimento mínimo de um ano. Entre as complicações, houve relato de dois casos de pseudartrose, três de necrose avascular e duas falhas do implante atribuídas a erro técnico.

Koukakis et $\mathrm{al}^{(17)}$, em trabalho prospectivo que incluiu 20 pacientes com fraturas em duas, três e quatro partes da classificação de Neer, tratados com essa mesma 
placa, apresentaram resultados satisfatórios, justificando que o uso desse implante fornece boa estabilidade em ossos osteopênicos, permitindo a mobilização precoce.

Mais recentemente, Moonot et $\mathrm{al}^{(18)}$ relataram resultados em 32 pacientes tratados com a placa Philos, mas em fraturas em três e quatro partes. Em 31 pacientes, houve consolidação clinica e radiográfica. Um caso evoluiu para necrose avascular, resultando a necessidade de uma hemiartroplastia. Outras complicações relatadas foram a consolidação viciosa em dois pacientes e o posicionamento errado da placa ou parafuso em três casos.

Em nossa casuística, todos os pacientes tiveram suas fraturas consolidadas, diferente da apresentada recentemente por Rose et al ${ }^{(19)}$, em que a consolidação ocorreu em $75 \%$ dos 16 pacientes tratados com a placa de sistema bloqueado. Em sua casuística, os quatro pacientes que evoluíram para pseudartrose apresentaram fratura em três partes associada à cominuição metafisária; em três casos, os pacientes eram tabagistas. Rose at $\mathrm{al}^{(19)}$ citam esse fator como de risco para retarde de consolidação e pseudartrose. Consolidação viciosa em varo ocorreu em três de nossos pacientes. Atribuímos essa complicação à má redução peroperatória antes da colocação do implante. É muito importante a obtenção da redução anatômica antes da colocação da placa bloqueada, visto que esse sistema não permite melhora da redução após a colocação dos parafusos bloqueados na cabeça umeral. Outro dado que consideramos importante é o posicionamento dos parafusos nas regiões com maior densidade trabecular óssea na cabeça umeral, que são as regiões central, inferior e posterior ${ }^{(20)}$. Quanto ao número de parafusos na cabeça umeral, não há trabalhos na literatura sobre esse assunto. Como na maioria de nossos pacientes foram colocados cinco parafusos, acreditamos que esse número permite boa segurança em relação à estabilidade. Uma desvantagem desse implante é o custo bem maior que o dos implantes convencionais.

Em nossa experiência, a osteossíntese com esse tipo de placa não revelou maiores dificuldades em relação a outros implantes utilizados previamente e o principal desafio foi a obtenção de redução anatômica, especialmente nas fraturas em três e quatro partes. A alta taxa de consolidação junto com a satisfação do paciente coloca esse método como mais uma boa alternativa de tratamento dessas fraturas.

\section{CONCLUSÃO}

A osteossíntese das fraturas da extremidade proximal do úmero com placas de ângulo fixo e parafusos bloqueados Philos demonstrou ser uma técnica com resultados funcionais satisfatórios e com baixo índice de complicações nesse grupo de pacientes.

\section{REFERÊNCIAS}

1. Habermeyer P, Schweiberer L. Fractures of the proximal humerus. Orthopade 1989;18(3):200-7.

2. Iannotti JP, Ramsey ML, Williams GR Jr, Warner JJ. Nonprosthetic management of proximal humeral fractures. Instr Course Lect. 2004;53:403-16.

3. Broos PL, Semon A. From unstable internal fixation to biological osteosynthesis. A historical overview of operative fracture treatment. Acta Chir Belg 2004;104(4):396-400.

4. Smith WR, Ziran BH, Anglen JO, Stahel PF. Locking plates: Tips and tricks. J Bone Joint Surg Am. 2007;89(10):2298-307.

5. Amstutz HC, Sew Hoy AL, Clarke IC. UCLA anatomic total shoulder arthroplasty. Clin Orthop Relat Res. 1981;(155):7-20.

6. Veado MAC, Moura ALL. Fraturas em duas e tres partes do umero proximal tratadas com sutura nao absorvivel. Rev Bras Ortop. 2007;42(10):333-42.

7. Checchia SL, Doneux SP, Miyazaki AN, Fregonese M, Silva LA, Lobo AC, et al. Avaliação do tratamento cirúrgico da fratura em duas partes do colo cirúrgico do úmero com placa PFS 80 R. Rev Bras Ortop. 2004;39(10):555-67.

8. Braman JP, Flatow EL. How to transition to percutaneous pinning for proximal humerus fractures. Tech Shoulder Elbow Surg. 2005;6:171-7.

9. Koval KJ, Gallagher MA, Marsicano JG, Cuomo F, McShinawy A, Zuckerman JD. Functional outcome after minimally displaced fractures of the proximal part of the humerus. J Bone Joint Surg Am. 1997;79(2):203-7.

10. Wanner GA, Wanner-Schmid E, Romero J, Hersche O, von Smekal A, Rrentz $\mathrm{O}$, et al. Internal fixation of displaced proximal humeral fractures with two onethird tubular plates. J Trauma. 2003;54(3):536-44.

11. Kristiansen B, Christensen SW. Plate fixation of proximal humeral fractures. Acta Orthop Scand. 1986;57(4):320-3.

12. Hintermann B, Trouillier $\mathrm{HH}$, Schafer D. Rigid internal fixation of fractures of the proximal humerus in older patients. J Bone Joint Surg Br. 2000;82(8):1107-12.

13. Meier RA, Messmer P, Regazzoni P, Rothfischer W, Gross T. Unexpected high complication rate following internal fixation of unstable proximal humerus fractures with an angled blade plate. J Orthop Trauma. 2006;20(4):253-60.

14. Siffri PC, Peindl RD, Coley ER, Norton J, Connor PM, Kellam JF. Biomechanical analysis of blade plate versus locking plate fixation for a proximal humerus fracture: Comparison using cadaveric and synthetic humeri. J Orthop Trauma. 2006;20(8):547-54.

15. Edwards SL, Wilson NA, Zhang L, Flores S, Merk BR. Two-part surgical neck fractures of the proximal part of the humerus. A biomechanical evaluation of two fixation techniques. J Bone Joint Surg Am. 2006;88(10):2258-64.

16. Bjorkenheim JM, Pajarinen J, Savolainen V. Internal fixation of proximal humeral fractures with a locking compression plate. A retrospective evaluation of 72 patients followed for a minimum of 1 year. Acta Orthop Scand. 2004;75(6):741-5.

17. Koukakis A, Apostolou CD, Taneja T, Korres DS, Amini A. Fixation of proximal humerus fractures using the PHILOS plate: early experience. Clin Orthop Relat Res. 2006;(442):115-20.

18. Moonot P, Ashwood N, Hamlet M. Early results for treatment of three-and fourpart fractures of the proximal humerus using the PHILOS plate system. J Bone Joint Surg Br. 2007;89(9):1206-9

19. Rose PS, Adams CR, Torchia ME, Jacofsky DJ, Haidukewych GG, Steinmann SP. Locking plate fixation for proximal humeral fractures: Initial results with a new implant. J Shoulder Elbow Surg. 2007;16(2):202-7.

20. Tingart MJ, Lehtinen J, Zurakowski D, Warner JJ, Apreleva M. Proximal humeral fractures: regional differences in bone mineral density of the humeral head affect the fixation strength of cancellous screws. J Shoulder Elbow Surg. 2006;15(5):620-4. 